\title{
APPLICATION OF TERAHERTZ RADIATION TO THE DETECTION OF CORROSION UNDER THE SHUTTLE'S THERMAL PROTECTION SYSTEM
}

\author{
Eric I. Madaras ${ }^{1}$, Robert F. Anastasi ${ }^{2}$, Stephen W. Smith ${ }^{3}$, Jeffrey P. Seebo ${ }^{4}$, James L. \\ Walker ${ }^{5}$, Janice K. Lomness ${ }^{6}$, Paul E. Hintze ${ }^{6}$, Catherine C. Kammerer ${ }^{7}$, William \\ P. Winfree ${ }^{1}$, and Richard W. Russell ${ }^{8}$ \\ ${ }^{1}$ NASA Langley Research Center, Nondestructive Evaluation Sciences Branch, Hampton, \\ VA 23681 \\ ${ }^{2}$ U.S. Army Research Laboratory, Vehicle Technology Directorate, AMSRD-ARL-VT-SM, \\ Nondestructive \\ Evaluation Sciences Branch, NASA Langley Research Center, Hampton, VA 23681 \\ ${ }^{3}$ NASA Langley Research Center, Metals \& Thermal Structures Branch, Hampton, VA \\ 23681 \\ ${ }^{4}$ Lockheed Martin, NASA Langley Research Center, Hampton, VA 23681 \\ ${ }^{5}$ NASA Marshall Flight Center, Damage Tolerance Assessment Branch, EM20 Huntsville, \\ AL, 35801 \\ ${ }^{6}$ NASA Kennedy Space Center, Corrosion Technology Laboratory, Kennedy Space Center, \\ FL, 32899 \\ ${ }^{7}$ United Space Alliance, Materials and Processing, Cape Canaveral, FL, 32920 \\ ${ }^{8}$ NASA Orbiter Project Office, MV7, Kennedy Space Center, FL, 32899
}

\begin{abstract}
There is currently no method for detecting corrosion under Shuttle tiles except for the expensive process of tile removal and replacement; hence NASA is investigating new NDE methods for detecting hidden corrosion. Time domain terahertz radiation has been applied to corrosion detection under tiles in samples ranging from small lab samples to a Shuttle with positive results. Terahertz imaging methods have been able to detect corrosion at thicknesses of 5 mils or greater under 1" thick Shuttle tiles and 7-12 mils or greater under 2" thick Shuttle tiles.
\end{abstract}

Keywords: Terahertz imaging, Hidden corrosion detection, NDE of thermal protection systems. PACS: 41.20.-q, 42.25.Dd, 42.25.Gy, 42.30.Va, 81.70.Ex, 89.20.Bb

\section{INTRODUCTION}

The United States Space Shuttle fleet consists of three shuttles with an average age of approximately 20 years. For most of a Shuttle's life, it is housed in an environmentally controlled building that protects it from corrosive elements. The Shuttles are exposed to potentially corrosive atmospheric elements while undergoing final closeout for missions at the launch pad and extreme conditions during ascent, orbit, and descent that may accelerate the corrosion process. Structural corrosion under the thermal protection system (TPS) is currently undetectable by NDE means and could progress undetected (without tile removal) and eventually result in the loss of local structural capability. Tile removal and replacement 
usually results in destruction of the tile and is a very expensive process for even just one tile. This has led NASA's Orbiter Program Office to seek an NDE inspection method to address this problem. The terahertz imaging process is of interest because it is sensitive to distortions in the metal surface such as might be caused by the generation of corrosion byproducts or by surface roughness and erosion that could accompany corrosion.

A series of steps was undertaken to examine the feasibility of inspecting for corrosion effects under Shuttle thermal insulation tiles with terahertz frequency, electromagnetic (EM), pulse echo NDE. The initial tests were done with lab samples that consisted of seventeen tiles mounted on an aluminum substrate. This represented a simple test without complications found in the actual orbiter. One of the tiles was removed and corrosion was induced by electrochemical means, and then the tile was replaced. Later tests were conducted on a large structure (which was used for training personnel in the method for removal and installation of tiles) that exhibited a high fidelity to actual orbiter construction. This training structure was not designed to have corrosion, but had been used for impact testing outdoors after the Columbia Accident Investigation, so the possibility of corrosion existed. In addition to tiles, measurements were also made on felt insulation samples which are another type of thermal protection system used on the shuttles. Finally measurements were started on the orbiter Atlantis.

\section{TEST ARTICLES}

The structure of the shuttle tile mounting system is roughly outlined in Fig. 1. The tiles are bonded to a felt-like pad that is known as a Strain Isolation Pad or SIP. The SIP is next bonded to the metal surface of the Shuttle. At the edges of the tiles, a strip of material called a filler bar is attached to the metal substrate, but not to the tile. These materials that are attached to the metal are porous, as are the tiles, and can hold moisture if exposed to water.

Laboratory measurements were made on several tiles to measure the electromagnetic wave velocities in the tiles. For reference, the velocities ranged from 2.74 to $2.81 \mathrm{x} 10^{8} \mathrm{~m} / \mathrm{s}$. (There are several varieties of tiles with different levels of density). Attenuation measurements were $3.06 \mathrm{~dB} / \mathrm{cm}$. These properties are useful in understanding imaging artifacts.

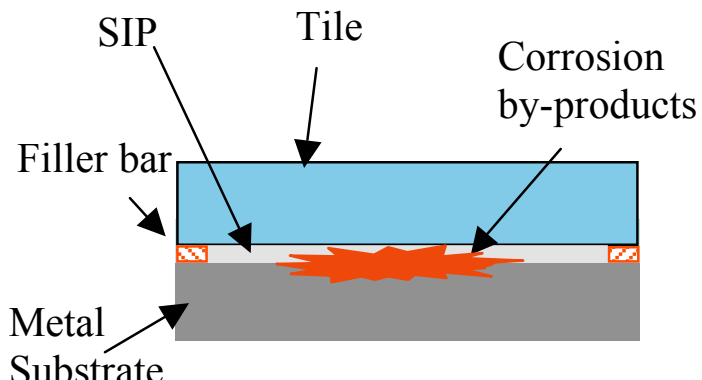

(a)
Presence of Corrosion

By-Products

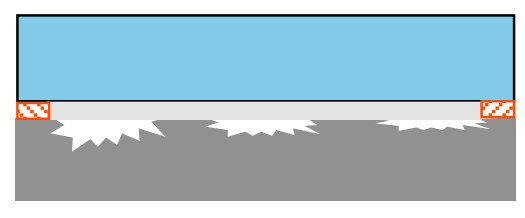

Surface Roughness Variations and Surface Erosion

Figure 1. Outline of the structure and the attachment of tiles to the metal substrate. Panel (a) represents a schematic diagram showing the presence of corrosion by-products and panel (b) is a schematic diagram showing the effects on the metal surface without corrosion byproducts. 


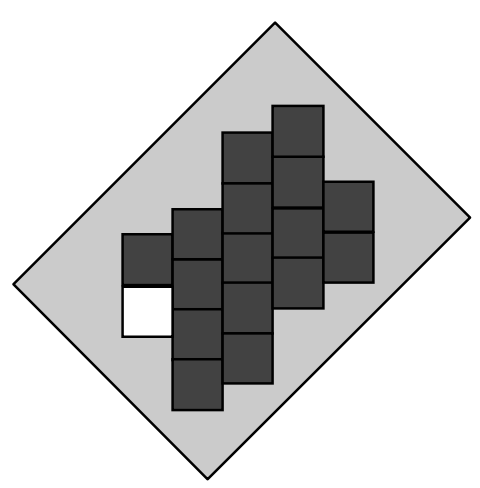

(a)

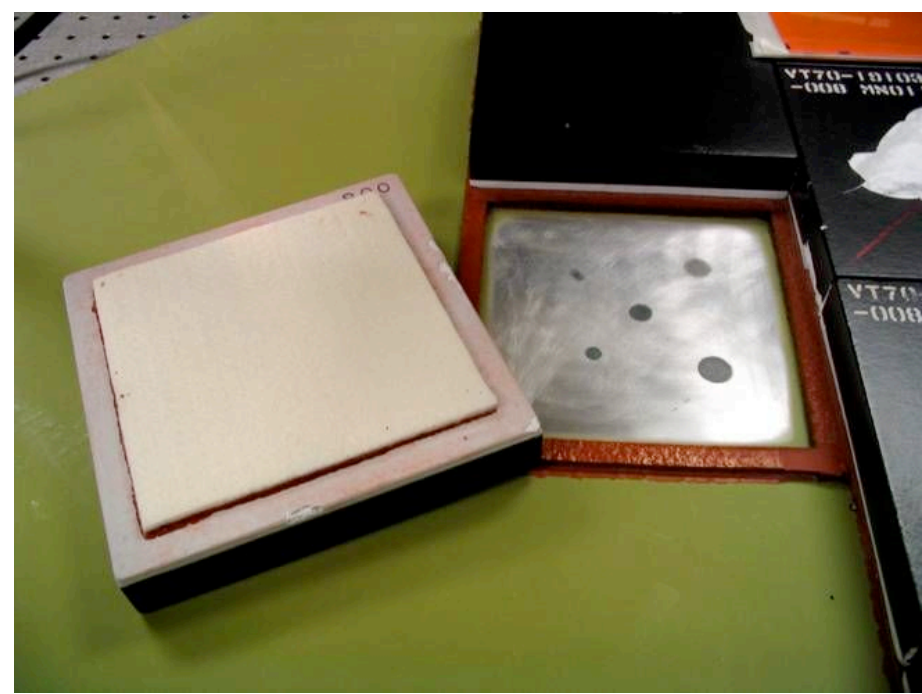

(b)

Figure 2. The first corrosion test article. (a) The lay-up of the 6" square tiles. (b) A photo of the corrosion pattern under the tile that was not attached.

Figure 2 shows features of the first corrosion test article. This test article had originally been developed for foam impact studies. The tiles were six inch square and approximately one inch thick. Figure $2 \mathrm{a}$ is the tile lay-up pattern. A tile on the lower left corner of the test article (Fig. 2a) was removed and left unattached for this first test as shown in Fig. 2 b. The substrate had the protective primer removed and five areas of corrosion were electrochemically created. The corrosion creating process involved placing a containment ring on the surface to hold a small amount of a salt solution. A current was passed through an electrode in the solution to generate the corrosion. The amount of current and the length of time were formulated to produce the desired depth and diameter of the corrosion. The corrosion products were left in place. A second tile was removed and a similar corrosion pattern was developed, but for that location, the tile was reattached and its location was not disclosed. For the exposed tile, the corrosion pattern in Fig. $2 b$ was: the top left spot was 0.25 " in diameter and had approximately 2.4 mils corrosion depth; the top right spot was 0.375 " in diameter and was too shallow to measure accurately with an optical microscope; the center spot was 0.375 " in diameter and the corrosion was 11.8 mils deep; the lower left spot was 0.25 " in diameter and the corrosion as 37.4 mils deep; and the lower right location was 0.50 " in diameter and 29.5 mils deep. A follow-up sample, similar in construction to the first test article, was manufactured and used as a blind test sample, i.e., it was not disclosed as to which tile locations were compromised or what corrosion existed under those tiles.

After the initial testing on these large articles, an additional small test sample was created that had 36 regions of corrosion on a 12" square aluminum plate which allowed for additional tests with thicker 6" square tiles or other thermal protection materials such as thermal felt coverings.

The final test article is the T35 training article (see Fig. 3), which was originally used to train technician staff to install thermal tiles and carbon-carbon leading edge panels. This article had been used for foam impact testing and accurately represents the actual Shuttle structure. No artificially induced corrosion was created in this article. 


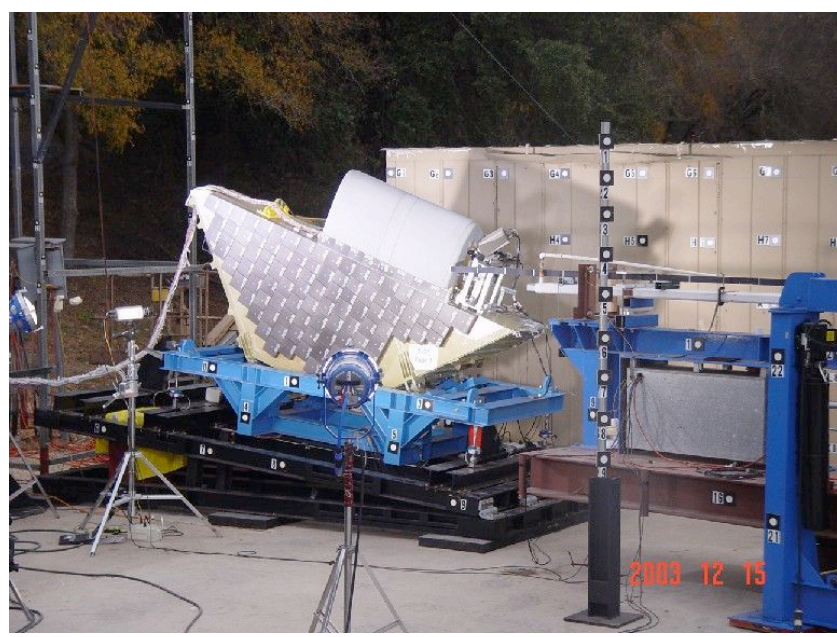

Figure 3. Photo of the T35 training article, which was also used for foam impact testing.

\section{RESULTS}

Initial results of imaging corrosion under tiles are illustrated in Fig. 4. Figure 4a shows a photograph of the corrosion area prior to the placement of the tile with the five regions of corrosion visible. Figure $4 \mathrm{~b}$ is a terahertz amplitude image of the metal substrate showing the corrosion areas without any intervening tile or SIP attachments. All five regions of corrosion are detectable. Figure $4 \mathrm{c}$ is a terahertz image of the substrate with the SIP and a 1" tile covering the corrosion areas. In Fig. 4c, the 11.7 mil and deeper corrosion areas were most detectable after integrating the data over the lower frequency ranges (75 $100 \mathrm{GHz}$ ). The 2.5 and shallower corrosion was not detectable due to the image noise induced by the SIP and the tile.

As a further test, this panel also has a tile that was covering a similar set of corrosion areas whose location was not disclosed. After scanning all the tiles for additional corrosion areas, it was readily evident which tile was covering the corrosion region. An image similar to the image in Fig. 4c was obtained.

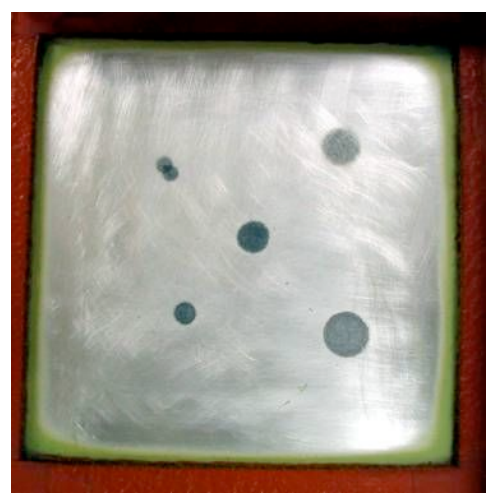

a)

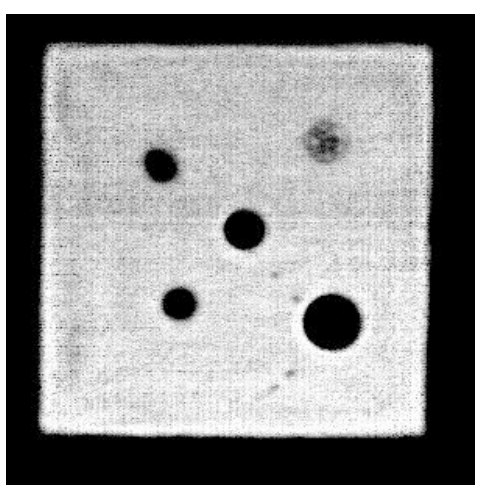

b)

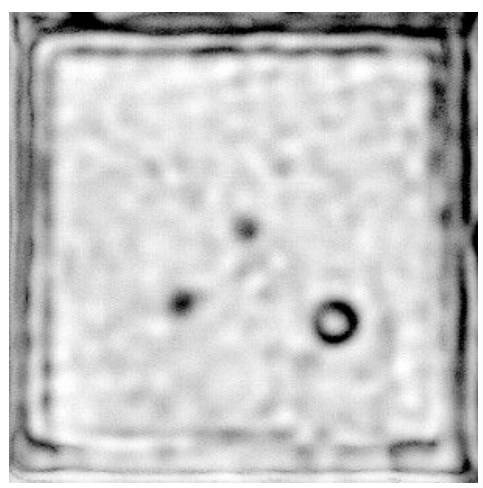

c)

Figure 4. Corrosion images of first test sample. a) Photo of corrosion on metal substrate. b) Terahertz image of metal substrate without tile. c) Terahertz image of metal substrate with intervening 1 " tile. 


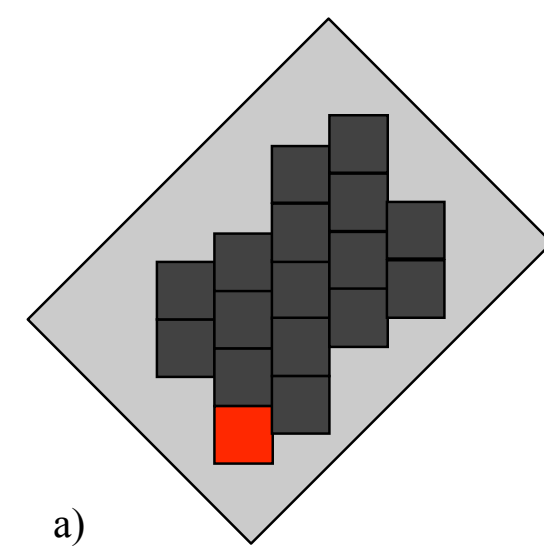

a)

Figure 5. Corrosion images of blind test sample. a) Tile lay-up of the test sample. The indicated square is the location of the tile that was ultimately identified as the tile with hidden corrosion. b) Terahertz image of metal substrate with corrosion hidden by the tile. c) Photograph of the corrosion areas with the depth of corrosion labeled.

For the next test, another 17 tile test panel which had man made hidden corrosion was scanned. This test article was considered a blind test article, and no information was provided prior to reporting the scan results. All scanned tiles produced images similar to each other except one, which is presented in Fig. 5. Fig 5a indicates the location of the tile with hidden corrosion. Figure $5 \mathrm{~b}$ shows the terahertz image. In this case, the general background pattern was different from the other tile scans and many areas of corrosion were evident. Figure $5 \mathrm{c}$ is a photograph of the corrosion areas, which was provided at the end of the testing. The depth in mils of each corrosion site is shown in the photo. Figure 6 is a graph that indicates qualitatively which corrosion sites were detectable and it plots those areas as a function of depth vs. the corrosion area's diameter. It should be pointed out that the determination of whether an area indicated corrosion or not depended both on the terahertz image in Fig. $5 \mathrm{~b}$ as well as on an investigation of the cross sectional terahertz

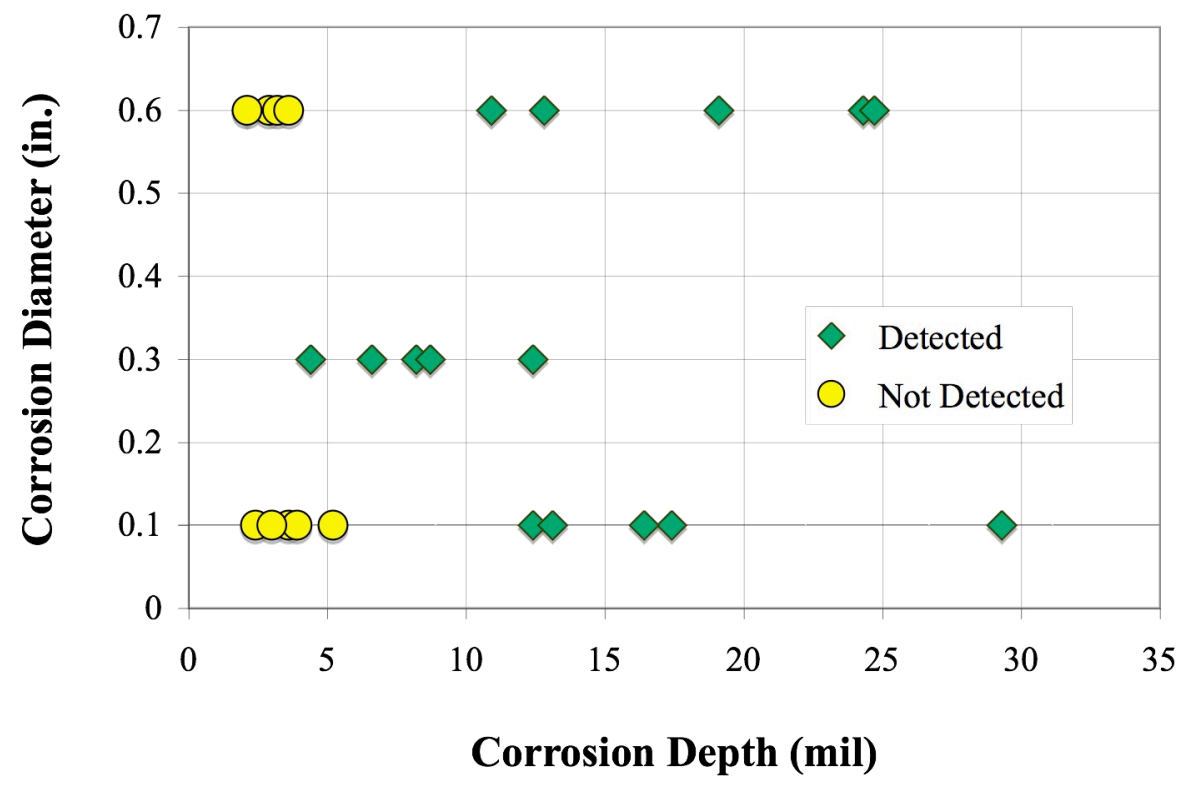

Figure 6. Graph showing the detection vs. corrosion geometry for a 1" tile. 


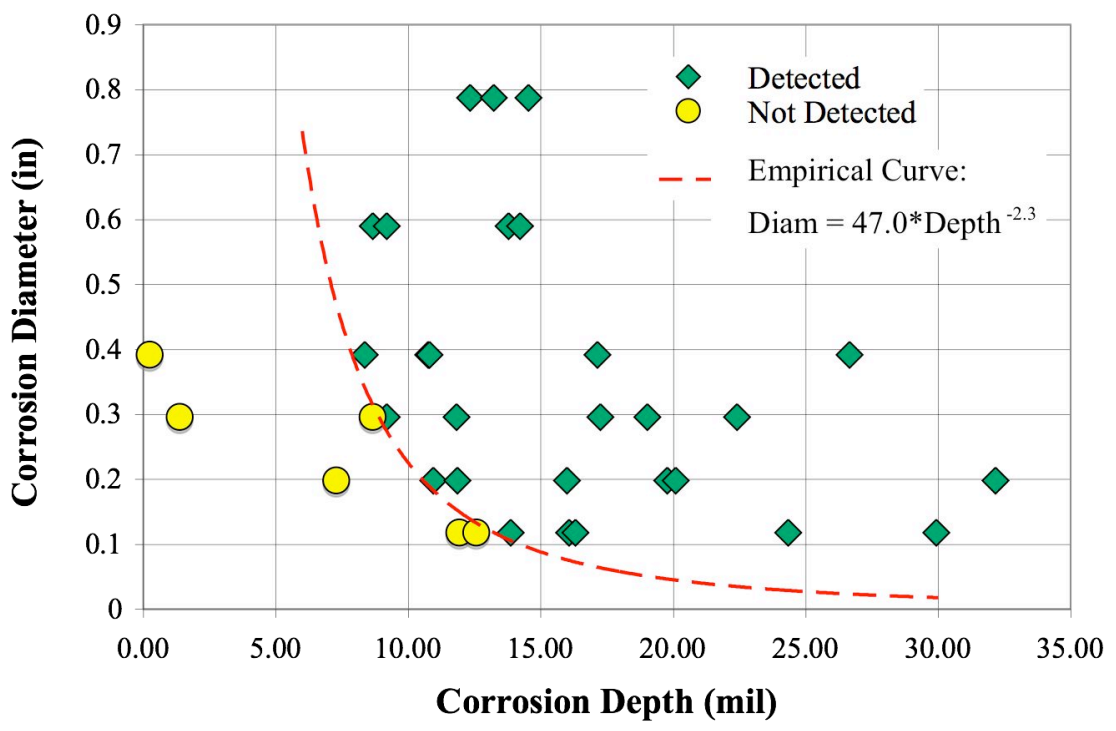

Figure 7. Graph showing the detection vs. corrosion geometry for a 2" tile.

image (a time slice of the data that was created in a manner similar to an ultrasonic B-scan) of the suspect area. It is evident that the detectability limit lies in the five mil range for detecting corrosion under one inch tiles.

As a follow up to these tests, a twelve-inch square test panel that had 36 areas of corrosion was manufactured. This panel then had tiles of additional thicknesses laid on top and scanned. Figure 7 is a graph showing the corrosion detectability under two inch tiles. In this case, the thicker tiles further degraded the signal for corrosion detection. The detectability of the corrosion areas is plotted against the corrosion depth vs. the corrosion area's diameter. A dashed line was generated that separated the detected corrosion areas from the undetected areas. The effect of diameter on the detectability is more evident in this graph. The detectability ranges from below seven mils at 0.3 in. diameter up to twelve mils at 0.1 in. diameter.

These tests illustrate the detection limits on flat plates. The orbiters' tile surfaces, which are more complex than flat plates, include curved and uneven surfaces, fasteners, joints and substructure. Additionally, the tiles may be marred or repaired in ways that affect terahertz images. To evaluate the effects of these conditions, further tests on a more representative sample were desired. It was decided that the next step was to apply terahertz imaging to a training article that had realistic structures similar to a shuttle's structure. Those measurements took place at NASA Kennedy Space Center and are shown in Fig. 8. This training article was approximately ten feet long and four feet high and was scanned in smaller regions. Figure 8 represents a collage of the images. This training article has numerous damaged tiles, which are easily seen in the terahertz image. Hy-Lock fasteners were readily evident, especially at the joints. The edge of the training article has a set of high density tiles which are seen as much darker in the image. In addition to the impact damaged tiles which look like either wide streaks in the terahertz image or as lighter areas, there were two repaired tiles which appear as dark dots in the image and are easily identified on the right hand side of Fig. 8. Figure 9a shows a terahertz close up of the training article's edge. Figure $9 \mathrm{~b}$ shows a cross-sectional view of one scan line from Fig. 9 a. At the right side of the cross-sectional image, there is a leveling compound under the 


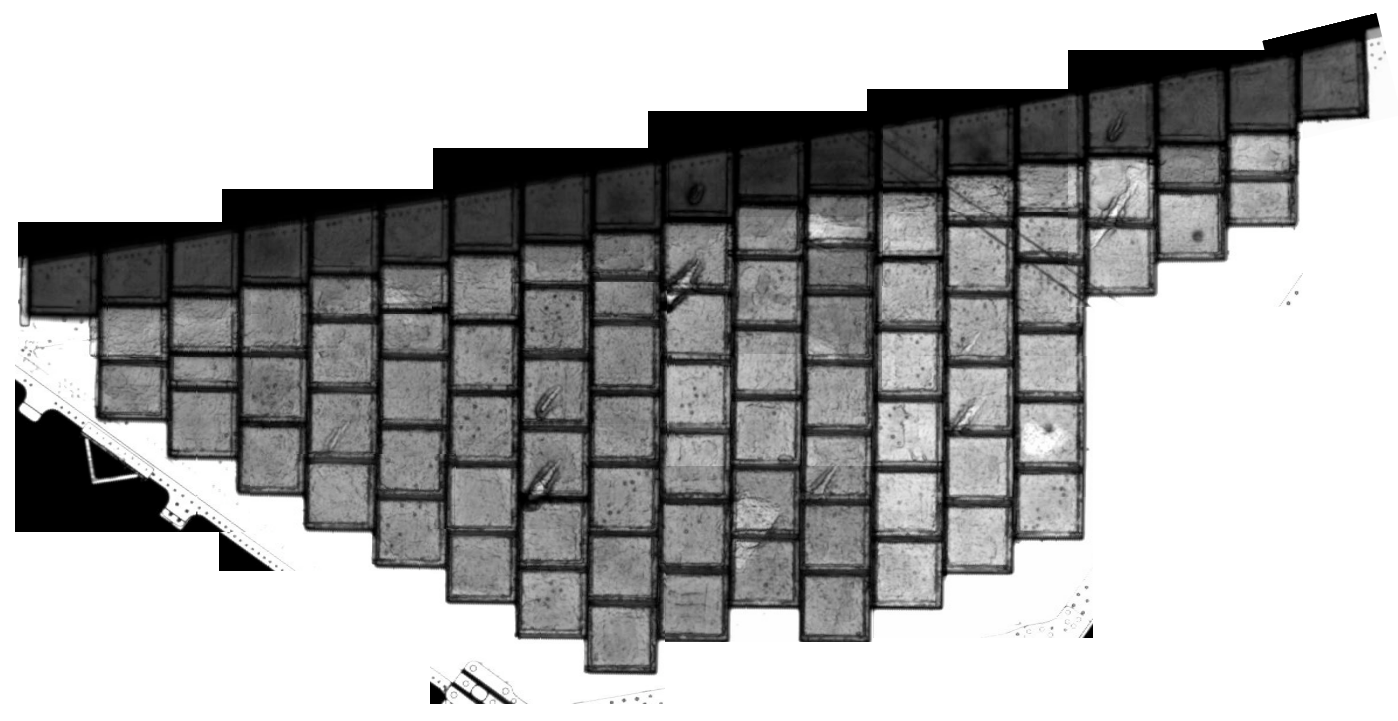

Figure 8. Terahertz image of the T-35 test article showing impact damage, subsurface structures, and tile repairs.

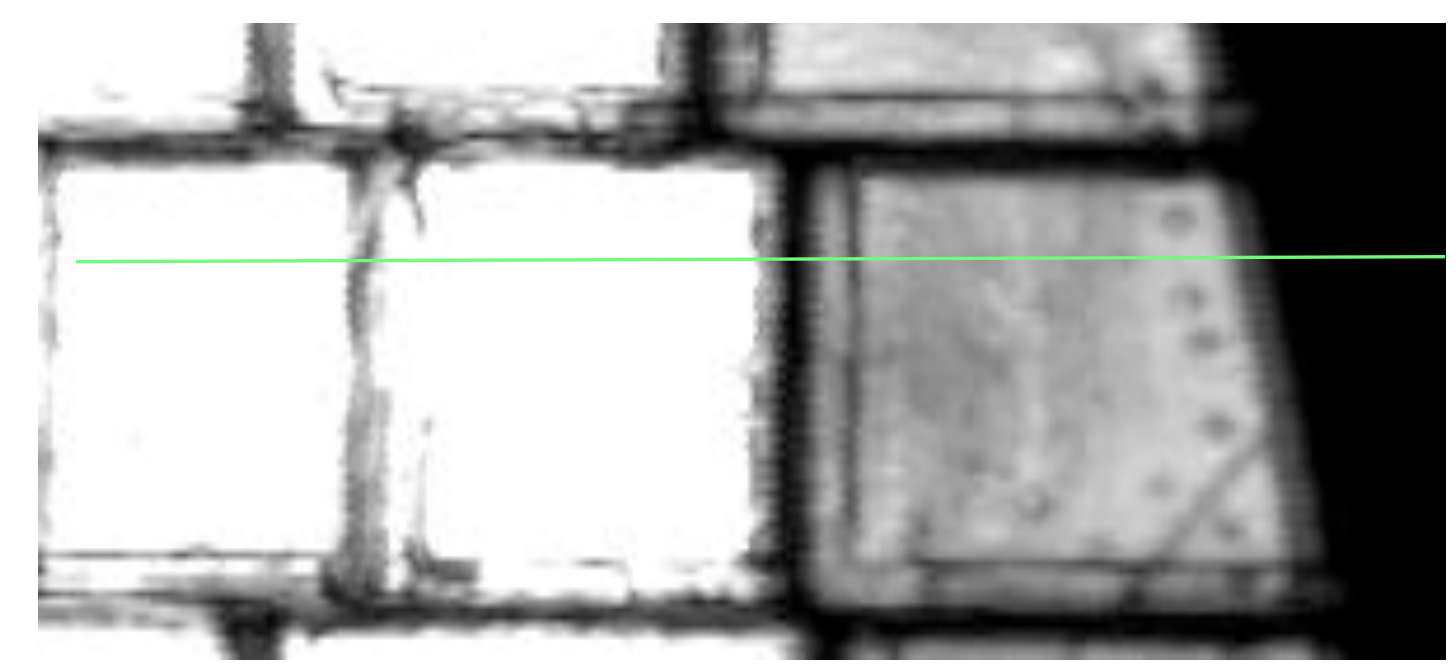

a)

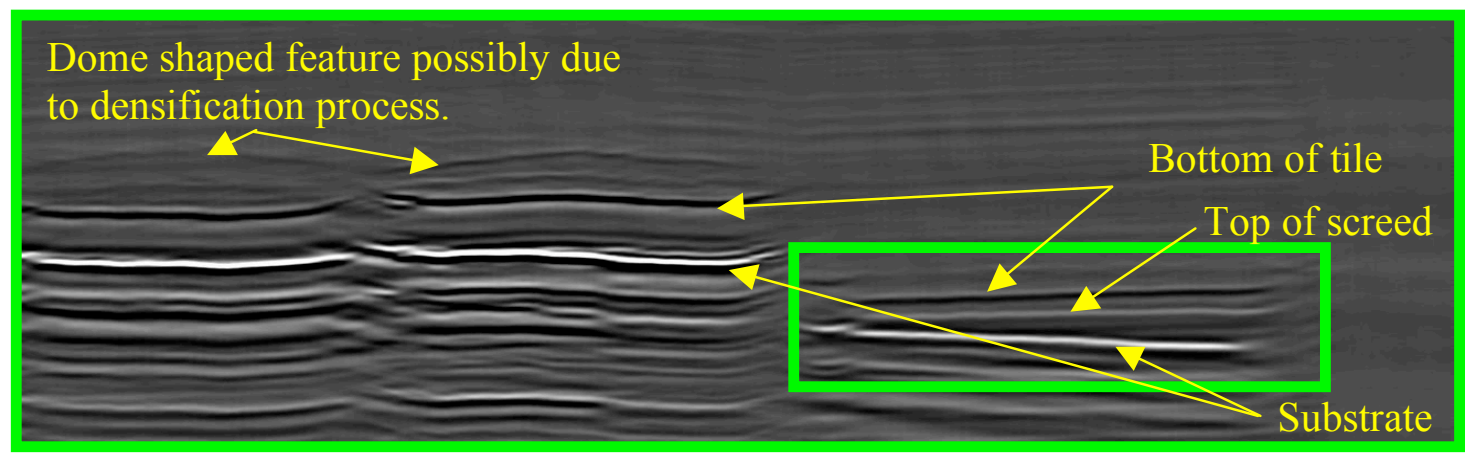

b)

Figure 9. Close up terahertz image from the T-35 training article. A) A terahertz image showing three tiles, one high density and two low density tiles. B) A cross section terahertz image showing the bottom of the tiles, the substrate, and presence of a leveling compound (called "screed"). 


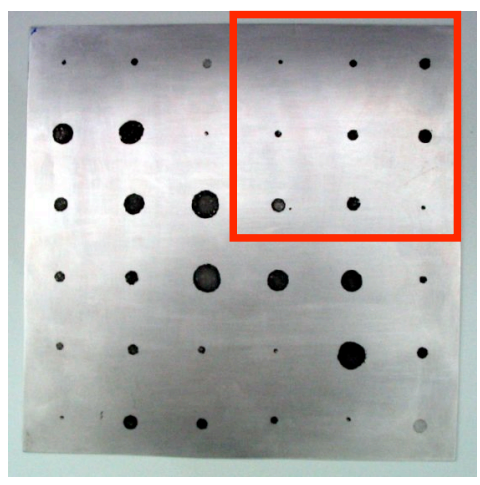

a)

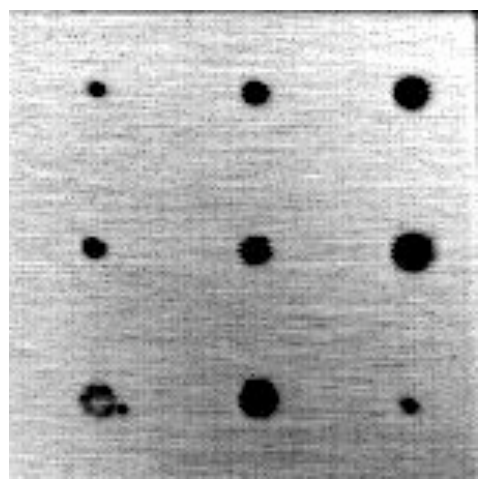

b)

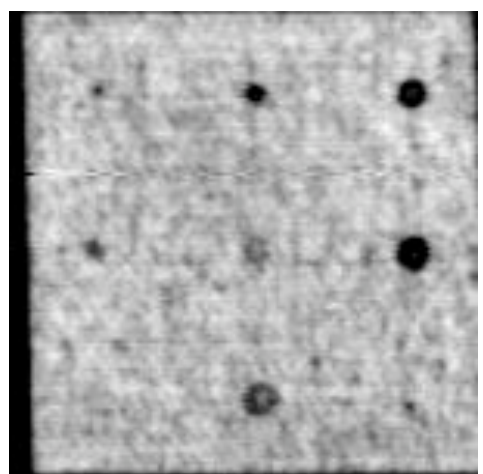

c)

Figure 10. Corrosion images of FRSI test sample. a) Photo of the corrosion sample. The indicated area shows the region scanned in images b) and c). b) Terahertz image of metal substrate without FRSI layer. c) Terahertz image of metal substrate with one FRSI layer.

edge tiles that was identified in the images. No areas of corrosion were detected by the terahertz imaging on this test article. To verify this fact, fifteen percent of the tiles were removed and no corrosion was seen.

In addition to tiles, there are other thermal protection materials that are used on the orbiters. One such material is called FRSI for Felt Reusable Surface Insulation. Figure 10 shows the results of scanning with a single layer of FRSI (approximately one half inch thick) on a plate with surface corrosion. Figure 10a is a photograph of the corroded test plate. Figure $10 \mathrm{~b}$ is a terahertz image of a corner of the corrosion test plate without any FRSI layers. Figure 10c is a terahertz image made with a single layer of FRSI covering the corrosions plate. It is clear that the FRSI insulation degrades the image, as several corrosion regions in Fig. 10b are no longer discernable in Fig. 10c. The degradation is roughly equivalent to that which occurred for two inch thick tiles.

\section{CONCLUSION}

Tests have been performed to evaluate the ability of EM radiation at terahertz frequencies to detect corrosion hidden under the thermal protection materials used on the Shuttles. The electromagnetic speed and attenuation properties of those thermal insulation materials were measured at terahertz frequencies. Subsequently, a series of measurements were made to determine the ability for corrosion detection under one inch thick shuttle tiles. Several measurements indicate that corrosion greater than five mils deep in the aluminum substrate is detectable under one inch thick tiles. Further measurements indicate that corrosion detection is limited to about seven to twelve mils under two inch thick tiles. Measurements on a relatively high fidelity structural mock up of the orbiter with tiles indicate that the terahertz EM waves had enough image resolution to identify numerous structures under tiles. Terahertz inspection of this test sample did not detect any areas of corrosion. Finally, tests on FRSI demonstrate that the detectability of finding corrosion under one layer of FRSI is roughly equal to results from scanning through two inch thick tiles. Further data has been collected during terahertz inspection of randomly sampled tiles on the orbiter Atlantis after it returned from its mission (STS-117) to the International Space Station. At this point in time, fifteen tiles were scanned for which no corrosion areas were detected and subsequently seven tiles were removed, verifying the lack of corrosion. 